\title{
Mortality Risk of Hypnotics: Strengths and Limits of Evidence
}

\author{
Daniel F. Kripke ${ }^{1,2}$
}

Published online: 13 November 2015

(C) Springer International Publishing Switzerland 2015

\begin{abstract}
Sleeping pills, more formally defined as hypnotics, are sedatives used to induce and maintain sleep. In a review of publications for the past 30 years, descriptive epidemiologic studies were identified that examined the mortality risk of hypnotics and related sedative-anxiolytics. Of the 34 studies estimating risk ratios, odds ratios, or hazard ratios, excess mortality associated with hypnotics was significant $(p<0.05)$ in 24 studies including all 14 of the largest, contrasted with no studies at all suggesting that hypnotics ever prolong life. The studies had many limitations: possibly tending to overestimate risk, such as possible confounding by indication with other risk factors; confusing hypnotics with drugs having other indications; possible genetic confounders; and too much heterogeneity of studies for meta-analyses. There were balancing limitations possibly tending towards underestimates of risk such as limited power, excessive follow-up intervals with possible follow-up mixing of participants taking hypnotics with controls, missing dosage data for most studies, and over-adjustment of confounders. Epidemiologic association in itself is not adequate proof of causality, but there is proof that hypnotics cause death in overdoses; there is thorough understanding of how hypnotics euthanize animals and execute humans; and there is proof that hypnotics cause potentially lethal morbidities such as depression, infection, poor driving, suppressed respiration, and possibly cancer. Combining these proofs with consistent evidence of association, the great weight of evidence is that
\end{abstract}

Daniel F. Kripke

dkripke1@san.rr.com

1 UCSD, 8437 Sugarman Drive, La Jolla, CA 92037-2226, USA

2 Scripps Clinic Viterbi Family Sleep Center, La Jolla, USA hypnotics cause huge risks of decreasing a patient's duration of survival.

\section{Key Points}

Most sleeping pills e.g., benzodiazepines and "Z" benzodiazepine agonists (like eszopiclone) are associated with early death, and they most probably cause serious increases in mortality.

Benzodiazepine agonist sleeping pills and diphenhydramine should not be prescribed for insomnia, except in long-term controlled trials and hospice care.

\section{Introduction}

The Cancer Prevention Study I of the American Cancer Society collected health questionnaires from over one million participants in the fall and winter of 1959-1960, obtaining $98.4 \%$ ascertainment of survival after 6 years. To examine associations of reported sleep durations and mortality, cross-tabulations were prepared controlling for reported 'insomnia' and use of 'sleeping pills,' as well as age, gender, and four major morbidities. Like Hammond's previous pilot analysis [1], the new analyses showed that mortality in both sexes had a U-shaped association with questionnaire-reported sleep duration, with the lowest risk for those reporting 7.0-7.9 h (coded as $7 \mathrm{~h}$ in this study) [2]. This association of sleep duration with mortality on 
6-year prospective follow-up was little influenced by control for reported insomnia or sleeping pill usage. Reported insomnia was only weakly and equivocally associated with mortality once hours of sleep and sleeping pill usage were controlled, but the presence or absence of reported insomnia or reported short sleep duration had relatively little impact on the association of sleeping pill usage, with about $50 \%$ increased mortality. In this early study, the phraseology of the sleep questions left much to be desired; particularly, lack of specification of which 'sleeping pills' were used 'often.' Because of the limitations of computer and software capacity at the time of analyzing data from over $1,000,000$ participants, the cross-tabulations for potential confounders were extremely rudimentary.

The sleep questions for the Cancer Prevention Study II (CPSII) were clarified in 1982, when new questionnaires were distributed to a new prospective sample of over 1.1 million participants. In particular, the focus was directed to 'prescription sleeping pills,' usage was reported in times per month, and prescription sleeping pills were separated from 'Librium' and 'Valium' as distinct categories. By the late 1990s, with powerful desktop computers becoming available, the coded and compiled data could be analyzed with Cox proportional hazards models simultaneously adjusting for 32 covariates [3, 4]. In the new study, the minimum mortality risk, though still coded as $7 \mathrm{~h}$, indicated that the best survival was with $6.5-7.4 \mathrm{~h}$ reported sleep. Some surprises in CPSII were that reported sleep durations $<6.5 \mathrm{~h}$ were less common and less associated with mortality risk than long reported sleep $\geq 7.5 \mathrm{~h}$. This might reduce motivations to drug patients to sleep for more than $7.5 \mathrm{~h}$. Reported insomnia predicted slightly (but significantly) improved survival, but greater sleeping pill use retained a dose-response association with decreased survival after adjusting for dozens of covariates [4]. Sleeping pill treatment was associated with much more risk than reported insomnia. Important limitations of the CPSII analyses included lack of definition of the particular prescription sleeping pills utilized and lack of consideration of the extent that participants maintained their prospectively reported use (or lack of use) of sleeping pills throughout the 6-year follow-up. Also, there was no correction for control participants who might have initiated sleeping pill consumption after the prospective baseline assessments. The sparse data concerning subsequent sleeping pill use after the baseline distinguished hypnotics users and controls were not taken into account. Even including 32 questionnaire-derived covariates in the Cox proportional hazards models, there were numerous conceivable confounders that might have been under-adjusted. In addition, adjusting for comorbidities that might have been caused by sleeping pills might have resulted in over-adjustments.
In the decades since these studies were done, although the issue of mortality associated with insomnia is still complex and controversial, dozens of studies have replicated the U-shaped association of reported sleep durations with mortality, and likewise, dozens of studies have replicated the association of hypnotics consumption with mortality. This review will update the list of studies relating mortality risk to hypnotics consumption, critique the limitations of those studies and their strengths, and point out the crucial remaining tasks.

\section{Methods}

By searching the last 30 years of PubMed publications for key words including both 'sedative hypnotic' and 'mortality,' 1700 titles were retrieved. The author reviewed each title along with the abstract and text as needed, in order to identify epidemiologic studies of hypnotics or hypnotics and anxiolytics that described follow-up mortality. Along with these publications, the systematic search for studies was supplemented by over 40 years of less formal reading of a much larger and more diverse sleep literature along with retrieval of relevant references listed in that literature. This located 36 epidemiologic studies analyzing whether use of hypnotics (with or without including sedative anxiolytics) predicted subsequent mortality, as listed in Table 1. Most of these studies focused on hypnotics rather than anxiolytics. Some nonEnglish language reports, reports not accessible at the University of California libraries website, and small studies in which the sample size or design was judged too inadequate were omitted. All of those deliberately omitted studies together constituted a small number without deliberate bias.

\section{Key Result: Use of Hypnotics is Certainly Associated with Increased Mortality}

All 34 of the studies that listed a mortality risk ratio, hazard ratio, or odds ratio found that the risk of hypnotic and anxiolytic use was $>1.0$ (in 33 studies) or 1.0 (in one small study). In other words, using hypnotics was followed by earlier deaths (Table 1). Of these, 24 individual studies found this positive association to be statistically significant $(p<0.05)$. This means that there were 24 studies that reported that hypnotic and sedative-anxiolytic use significantly predicted early mortality. There were no studies reporting any evidence of a protective effect of hypnotics; that is, there were no studies with a risk ratio of $<1.0$ (Table 1). 
Table 1 Epidemiologic studies concerning the mortality risk of hypnotics and anxiolytics

\begin{tabular}{|c|c|c|c|c|}
\hline Study & Participants $(N)$ & Years & Risk, OR or $\mathrm{HR}^{\mathrm{a}}$ & Significance \\
\hline Kripke et al., 1979 [2] & 823,065 & 6 & $1.13-1.57$ & $p<0.001$ \\
\hline Rumble and Morgan, 1992 [62] & 577 & 5 & $1.19-1.24$ & $p=0.027-0.275$ \\
\hline Isacson et al., 1992 [63] & 19,926 & 8 & $>1$ & Mostly significant \\
\hline Thorogood et al., 1992 [64] & 112 & 3 & $3.7-12.0$ & $p<0.05$ \\
\hline Brabbins et al., 1993 [65] & 1063 & 3 & Not given & NS \\
\hline Merlo et al., 1996 [66] & 498 & 10 & 1.0 & NS \\
\hline Hays et al., 1996 [67] & 3962 & 4 & 1.03 & NS \\
\hline Sundquist, et al., 1996 [68] & Unclear & 10 & Not given & $p<0.001$ \\
\hline Merlo et al., 2000 [69] & 491 & 10 & 1.2 & NS \\
\hline Kojima et al, 2000 [12] & 5322 & 12 & 1.62 & NS \\
\hline Kripke et al., 1998 and $2002[3,4]$ & $1,116,936$ & 6 & $1.24-1.25$ & $p<0.05$ \\
\hline Hedner et al., 2002 [70] & 1211 & 5 & 1.65 & $p=0.01$ \\
\hline Mallon et al., 2002 [71] & 1870 & 12 & 6.4 and 3.8 & $p<0.05$ \\
\hline Ahmad and Bath, 2005 [72] & 1042 & 15 & 1.013 & NS \\
\hline Phillips and Mannino, 2005 [73] & 13,563 & 6.3 & 1.4 & NS \\
\hline Lack et al., 2006 [74] & 2087 & 13 & 1.12 & $p=0.001$ \\
\hline Fukuhara et al., 2006 [75] & 5041 & 5 & 1.27 & $\mathrm{p}=0.04$ \\
\hline Hausken et al., 2007 [76] & 14,951 & 18 & 1.6 & $p<0.05$ women \\
\hline Hoffmann et al., 2007 [77] & 7658 & 3 & $\sim 1.5$ & $p=0.002$ \\
\hline Hublin et al., 2007 [78] & 21,268 & 22 & 1.7 & $p<0.05$ \\
\hline Winkelmayer et al., 2007 [79] & 3630 & $2-4$ & 1.15 & $p<0.05$ \\
\hline Mallon et al., 2009 [15] & 3523 & 20 & 3.285 & $p<0.001$ \\
\hline Belleville, 2010 [16] & 14,117 & 12 & 1.36 & $p<0.05$ \\
\hline Rod et al., 2010 [80] & 16,989 & 19 & $1.07-1.30$ & $p<0.05$ and NS \\
\hline Gisev et al., 2011 [81] & 1845 & 9 & 1.01 & NS \\
\hline Kripke et al., 2012 [10] & 34,205 & 2.5 & $3.60-5.32$ & $p<0.001$ \\
\hline Hartz and Ross, 2012 [82] & 148,938 & 8 & 1.14 & $p<0.05$ \\
\hline Obiora et al., 2012 [13] & 34,661 & 2.8 & $1.42-2.17$ & $p<0.001$ \\
\hline Jaussent et al., 2013 [5] & 6696 & 8.9 & $1.03-1.11$ & NS \\
\hline Chen et al., 2013 [17] & 4064 & 7 & 1.37 & $p<0.05$ \\
\hline Weich et al., 2014 [9] & 104,145 & 7.6 & $2.08-3.32$ & $p<0.001$ \\
\hline Frandsen et al., 2014 [83] & 2588 & 7 & $1.27-1.33$ & $p<0.005$ \\
\hline Weisberg et al., 2015 [84] & 64,602 & 1 & $1.26-1.33$ & $p=0.004$ \\
\hline Pinot et al., 2015 [85] & 2350 & 2.8 & 1.08 & NS \\
\hline Neutel and Johansen, 2015 [14] & 907,780 & 2 & 2.3 & (95\% CI 2.2-2.4) \\
\hline Nakafero et al., 2015 [86] & 25,887 & 1.97 & $1.58-2.78$ & $p<0.02$ \\
\hline
\end{tabular}

Expanded with permission from the Ref. [10] supplement NS not significant

${ }^{a}$ When provided in the study, the mortality risk ratio, odds ratio, or hazard ratio for hypnotics users is listed. An association of hypnotics use with reduced mortality would be represented as a risk ratio $<1.0$

\section{Limitations}

\subsection{Power}

The power of the study influenced the chances of finding significant association: all 14 reported associations with a sample size $>14,000$ were significant, but 10 of the 22 studies with sample size $<14,000$ found a similar trend but not a significant association. Most of the smaller studies failing to find significance in the association were among the earliest 15 published (2005 or earlier).

\subsection{Excessive Follow-Up Interval}

An over-lengthy interval of prospective mortality followup from the baseline determination of hypnotic use was an 
additional factor that seemed to influence the outcomes. Only 4 of 22 studies that analyzed follow-up of 8 years or less failed to report a significant association, but 6 of 14 studies analyzing follow-up intervals $>8$ years failed to find significant association. One explanation of this is obvious. Participants who may be utilizing hypnotics at baseline do not necessarily continue to use them for many years. For example, in a study following patients' mortality for up to 12 years, Jaussent et al. reported that less than one third of those who had taken a hypnotic at baseline or at 2 , 4, and 8-year follow-ups were taking a hypnotic at all four assessments (online comment doi:10.1186/1741-7015-11212 [5]). Moreover, a percentage of patients $69 \%$ as great as those taking a hypnotic at baseline initiated hypnotic usage after baseline during the first 8 years of the 12-year follow-up. Therefore, in the 12-year maximum follow-up, it appears that the number of study patients commencing hypnotic usage after baseline might be almost as great as those using a hypnotic at baseline. Were baseline usage the prospective criterion for dividing hypnotic users from controls, it may be seen that over 12 years, the actual hypnotic consumption of the control group would more and more approximate that of the baseline hypnotic users. If a study separates hypnotic users and controls prospectively at baseline without monitoring hypnotic consumption during follow-up, the longer the study, the more strongly the hypnotic consumption of 'users' and 'controls' is likely to overlap. Methods of adjustment such as time-dependent proportional hazards models, when feasible at all, have unknown adequacy for adjusting these usage factors. Usage overlap of hypnotic-user cases and their controls tends to weaken the contrast between hypnotic users and controls, leading to underestimation of the true hypnotic-associated risk ratio. The two large American Cancer Society studies both had this limitation, tending towards underestimation of risk [2-4].

\subsection{Confounding Drugs with Different Indications}

For perhaps a century, the most popular hypnotics have been drugs that augment the action of ligand-gated anion channels, usually called GABA receptors. GABA acts to open chloride channels that generally lead to intracellular hyperpolarization and consequent inhibition of neuronal firing. Although ethanol and barbiturates react in parallel at other sites on GABA receptors, for decades the predominant hypnotics have been benzodiazepines and benzodiazepine agonists acting on benzodiazepine binding sites on GABA receptors and tending to amplify GABAergic effects $[6,7]$. To oversimplify, these drugs tend to shut down brain activity. Histamine and antihistamines may also act on GABA receptors [8], raising a question whether antihistamines with hypnotic properties might also act in part through effects on GABA receptors. Some of the same drugs have hypnotic and anxiolytic properties, as well as muscle-relaxant and anticonvulsant effects. Some of the most important differences among benzodiazepine agonists have to do with the speed of absorption and the metabolic half-life of the drug and active metabolites. For example, a drug such as zaleplon is quickly absorbed but acts for only $1-3 \mathrm{~h}$ before it is mostly inactivated. A drug such as zolpidem or triazolam is rapidly absorbed and acts for most of the night, but rapid drug metabolism may produce early awakening or daytime hyper-arousal or anxiety, whereas a drug such as lorazepam may be somewhat more slowly absorbed and long-acting enough to sedate during the day after bedtime administration. Drugs such as diazepam and flurazepam, with their active metabolites, persist in their actions for several days and accumulate (the degree of accumulation depending on metabolic processes that are often slowed in old age). Chlordiazepoxide persists in the body for weeks.

A wise physician may choose a different hypnotic for a patient who has trouble falling asleep compared with a patient who primarily complains of early awakenings. Longer acting drugs may be selected for anxiolytic effects at the risk of accumulating daytime sedation, and drugs intended as anxiolytics are often given in the day rather than at night. Thus, the adverse effects will depend both on the drug and the indication. Because benefits and risks certainly vary according to time of administration and time of day, it may be irrational to combine GABA agonists with widely varying kinetics in the same risk analysis. A short-acting drug given at bedtime may have strong effects on respiratory depression when combined with a narcotic but little effect on next-day driving. A tranquilizer given in the morning will have more effect on driving but less effect on nocturnal respiration.

Only a minority of the studies in Table 1 ascertained the actual drugs being used as sleeping pills. Many patients who take a drug at night for sleep have no idea of its pharmacologic indications, so questionnaire data are often extremely nonspecific as to the drug taken. Some of the studies may have included non-prescription sleeping pills that have historically contained antihistamines such as diphenhydramine, but may have also contained non-steroidal anti-inflammatory or scopolamine-like drugs. Most of the epidemiologic studies of hypnotics to date have been confounding short-acting hypnotics and long-acting anxiolytics, along with antihistamines, nonsteroidal anti-inflammatories, antidepressants, and neuroleptics, usually in unknown proportions. Such confounding no doubt impacts the reported risks in ways that are quite unknowable. Although a patient may report any variety of drugs as sleeping pills, many of the drugs have no currently recognized medical indication for sleep at all, so that whether 
a drug with an other-than-hypnotic indication does or does not influence mortality risk, its inclusion obscures precise focus on the risks of true hypnotics.

In large electronic records studies [9, 10], it has appeared that all benzodiazepine agonists examined (including ' $\mathrm{Z}$ ' drugs) are associated with mortality risks. Diphenhydramine and possibly other similar antihistamines have also been implicated in mortality risks $[10,11]$. Clear epidemiologic studies of mortality risks seem unavailable for trazodone, doxepin (especially low-dose doxepin), and melatonin when used as hypnotics.

\subsection{Unknown Dosages Consumed}

Very few of the studies listed in Table 1 used pharmacy records of what quantities of hypnotics were actually dispensed, and few studies had data on the numbers of doses prescribed over the interval of follow-up. Needless to mention, dispensed hypnotics often sit in the medicine chest with most doses unused, but other doses may be shared with relatives or may even be resold more widely. Having at best only rough estimates of the quantities of drugs that patients in the hypnotics-taking groups consumed, even the best epidemiologic studies of hypnotic risks are only poorly able to examine dose response. Of course, even when the defined daily dosage (DDD) of a drug a study participant has consumed can be estimated, drug effects are known to vary widely by age, gender, and body mass of the patient, metabolic considerations (especially CYP3A enzyme inhibitor effects), and drug combinations, so the implications of dosages are still quite controversial.

\subsection{No Formal Meta-Analysis}

The reader who understands what meta-analyses can do will immediately understand that with 33 out of 34 epidemiologic studies pointing in the same direction, and with high levels of statistical reliability in many of the single studies, meta-analysis is not necessary to be assured of the statistical significance of the hypnotics-mortality association. At the same time, the studies listed were exceptionally heterogeneous in the nature of the drugs studied; knowledge of the actual compounds studied and the quantities actually consumed by research participants; the numbers of participants; characteristics of participants including age, gender, health status, and other-risk exposures; the durations of follow-up; and the statistical details, especially adjustments of potential confounders. There are so many important sources of heterogeneity between studies that the author sees no satisfactory method of selecting homogeneous subgroups of studies for meta-analyses. Meta-analysis would only produce additional risks of confounding due to study selection. Several of the studies provided such statistically firm results in themselves that combination with other studies would be superfluous. Three such studies will be discussed at some length, each of which used electronic prescription records to unfold the prescriptions patients received for specific drugs during follow-up and to some extent before the baseline as well.

Weich et al. examined a representative population sample from the UK General Practice Research Database [9]. Hypnotic users had received over a 4-year interval at least two prescriptions for a benzodiazepine (both hypnotics and anxiolytics), $\mathrm{Z}$ hypnotic, or other hypnotic, and had not received such drugs prior to study initiation. Considering patients who had received a study drug in the first year of recruitment only versus matched hypnotic-free controls, fully adjusted hazard ratios for hypnotic usage predicting mortality ranged from 1.0 (no exposure, the reference) to 2.55 (95\% CI 2.42-2.69) for 1-30 DDDs, 3.78 (95\% CI 3.54-4.04) for 31-60 DDDs, 4.19 (95\% CI 3.84-4.58) for 61-90 DDDs, and 4.51 (95\% CI 4.22-4.82) for $>91$ DDDs. All of these mortality associations for hypnotics exposures were highly significant $(p<0.001)$. The hazard ratios were highest among patients receiving benzodiazepines only and somewhat lower among patients receiving $\mathrm{Z}$ drugs only or other hypnotics only, but all demonstrated significant dose response. These hazard ratios were fully adjusted for sex, age, sleep disorders, anxiety or other psychiatric disorders, and many medical morbidities such as heart disease, diabetes mellitus, and cancer. Comorbidities were considered prospectively, and concurrent comorbidity was considered separately. Either prospective or concurrent covariate adjustment reduced the hazard ratios rather little. The modest impact of either prospective or concurrent extensive full adjustment for comorbidities and other confounders suggested that there was little confounding by indication (that is, confounding because study drugs were selectively given to patients because they were already perceived as ill.) The investigators suggested "that much of the excess mortality risk arises early in the period of drug use but remains statistically significant even after discontinuing the drugs." Nevertheless, the investigators noted that residual confounding could not be excluded; for example, due to confounders that could not be adequately measured.

Kripke et al. examined mortality among patients of a large rural US managed-care organization using electronic medical records of medical diagnoses, a cancer registry, and prescriptions [10]. Patients who received a hypnotic were matched on age, gender, smoking, and various prior and concurrent morbidities with controls not receiving hypnotics and followed for an average of 2.5 years. The association of hypnotics with increased mortality was highly significant $(p<0.001)$. Zolpidem and temazepam 
were the hypnotics most commonly prescribed. Only indicated hypnotics prescribed at bedtime were included in this analysis. As in the Weich et al. study [9], a doseresponse relationship with hypnotic prescribing was noted, but much of the mortality hazard was associated with low doses and/or short-term prescribing. Considering the many design differences between these two studies, the hazard ratios were surprisingly similar. As in the UK study, extensive and varied efforts to adjust for confounding by intention to treat and to isolate various comorbidities suggested that confounding had only a small impact on a study done in this way. However, the authors admitted that some small element of confounding was likely.

Obiora et al. examined pneumonia mortality in 4964 cases and 29,697 controls also selected from the UK primary care patient database [13]. Using the Charlson's comorbidity index to adjust for comorbidities, as compared with controls, fully adjusted long-term mortality hazard ratios were 1.42 (95\% CI 1.21-1.67) for current benzodiazepine use, 1.49 (95\% CI 1.19-1.85) for recent benzodiazepine use, and 1.24 (95\% CI 1.09-1.41) for past benzodiazepine use. There were few zopiclone data, but for current use, the adjusted hazard ratio was likewise significant at 2.17 (95\% CI 1.17-4.06).

The relatively high and highly significant hazard ratios observed in these studies indicate the value of using electronic databases to identify hypnotic prescriptions, comorbidities, and associated mortality. These studies demonstrate an association of hypnotic use with subsequent mortality risk that needs no meta-analysis for confirmation. Although these studies made extensive attempts to avoid confounding and to counteract it, using highly developed databases, none was able to claim total freedom from risks of confounding factors. As a consequence, the causality of hypnotics causing excess mortality remained somewhat open to challenge.

It is worth observing that these three studies yielded among the highest hazard ratios of the 34 studies listed in Table 1. Probably-important factors in the relatively high hazard estimates were exclusion or limitation of patients using hypnotics before the baseline observations; the relatively brief follow-ups enabling ongoing monitoring of hypnotic prescriptions among both those classed as hypnotic users and matched controls, thus minimizing intermingling of the groups; the availability of dose-response data; and the quality of information about comorbidities as well as smoking and BMI.

\subsection{Confounding by Indication}

To show a large and statistically very significant association between hypnotics and the subsequent risk of early mortality is not absolute proof that the hypnotics cause that mortality.

A recent publication presented evidence that hypnotic prescribing is increased for a few months before death for patients possibly manifesting terminal illnesses [14]. This was interpreted as confounding by indication (i.e., the terminal illness caused the hypnotic usage). Such terminal prescribing may be appropriate, since hypnotics might be useful hospice agents in situations where prolonging a patient's life is hopeless and the amnesic and anxiolytic effects of hypnotics seem desirable. However, the same data set showed that 2 years before death, usage was already elevated among patients who would die, but roughly parallel increases in hypnotic use were observed among patients who did or did not die in the first year of the 2-year observations [14]. This is consistent with hypnotics causing terminal conditions, but the increase in hypnotic usage in the year before death also seemed consistent with hypnotics rapidly causing terminal conditions. Such data by themselves do not at all argue that the association of hypnotics with excess mortality can be entirely attributed to confounding by indication, and unfortunately, the study did not control for comorbidities.

If a physician prescribes a hypnotic for insomnia or for depression with insomnia, commentators worry that it is the insomnia and not the hypnotic that causes the associated mortality. However, insomnia and depression are probably not important sources of confounding, since several studies found that adjusting for these comorbidities made little change in the hazard ratios for hypnotics $[4,15-17]$.

A physician may prescribe a hypnotic because of a suspicion that the patient needs to sleep better, even when no complaint of insomnia is present or no diagnosis of a sleep disorder happens to be recorded. A physician might believe (without it being an accepted indication) that hypnotics are good for hypertension or heart disease or obesity or ulcers, etc., and thus the hypnotic prescription could be a sign of diverse dire disorders. If these other disorders are accurately diagnosed, then these confounding sources of mortality risk can be managed by statistical adjustments for comorbidities (see Figs. 1, 2, 3). If no prospective medical diagnosis is made of the disorder being treated by the hypnotic (for which statistical adjustment would be possible), but the physician felt through empathy or the bedside manner or something more mysterious that there was a need for a hypnotic, then the confounding indications probably cannot be adequately managed statistically.

There is evidence for much mysterious prescribing of hypnotics, of which the US National Ambulatory Medical Care Survey provided useful weighted estimates from a 


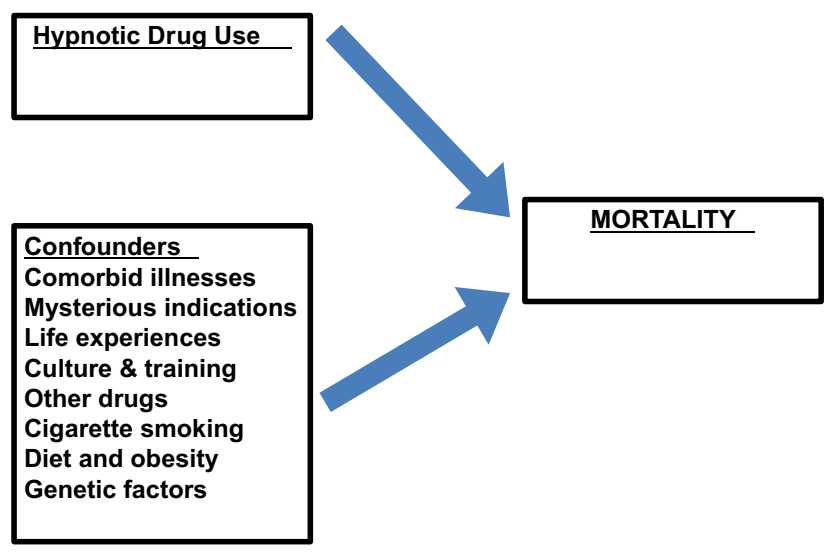

Fig. 1 A causal model in which hypnotic drugs and various potential confounders have completely independent effects on mortality. A Cox proportional hazards model should easily separate these independent covariates of mortality

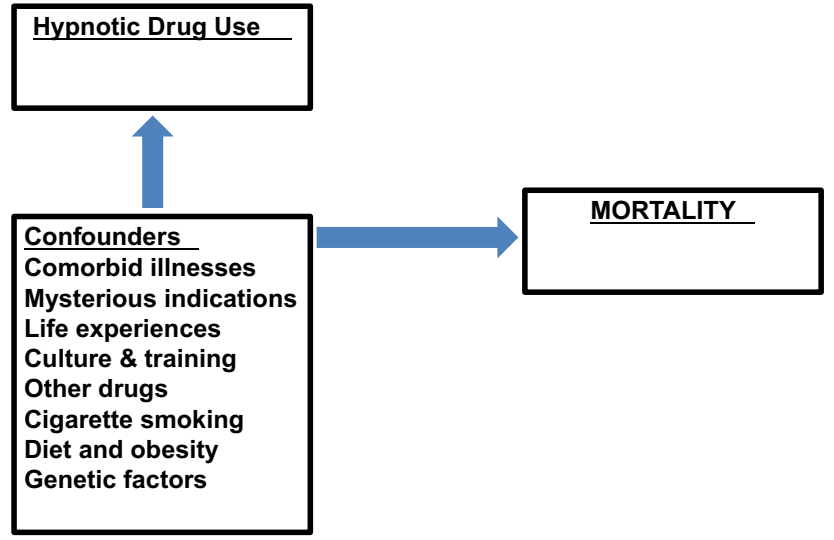

Fig. 2 A causal model in which confounders influence hypnotic use and independently influence mortality, but hypnotic drugs are intended to treat the primary confounders and have no direct influence on mortality. In this case, there will appear to be an association between hypnotic drug use and mortality that is completely confounded by intention and wholly attributable to the correlations of both hypnotic use and mortality with the confounders. However, the proportional hazards model could reject hypnotic drug use as a hazard, since adjusting for the confounders would better explain the variance, unless certain confounders were inadequately or imprecisely specified

national sample [18]. In 2009, there were an estimated 5.2 million office visits in which insomnia was a stated reason for the visit and a total 10,224,000 visits for any sleep disturbance (including disturbances for which prescribing a hypnotic would not be appropriate, such as most cases of sleep apnea). A sleep-related diagnosis (not just insomnia; e.g., includes sleep apnea) was listed in 15.2 million visits, but there were 23.3 million visits upon which a prescription was given for a hypnotic medication FDA-indicated for insomnia. Expanding the enumeration to include such medications as alprazolam, clonazepam,

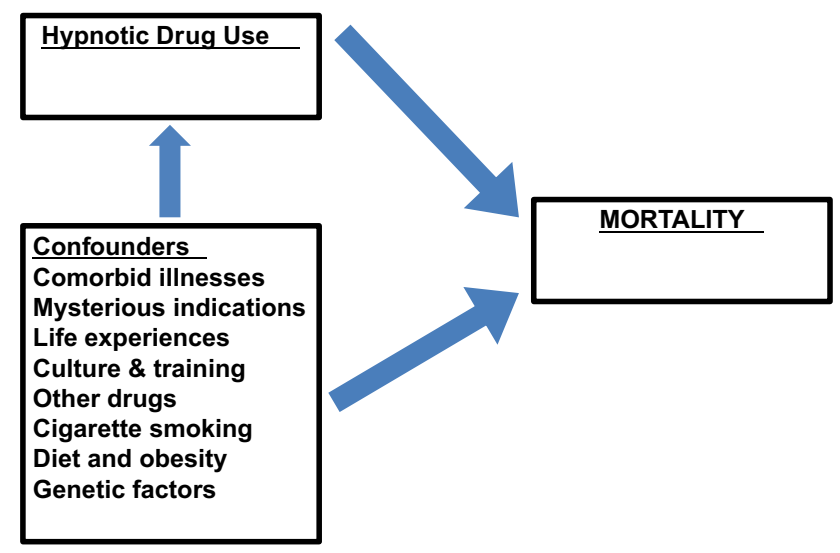

Fig. 3 A causal model in which confounding factors influence mortality directly and also indirectly through the pathway mediated through confounder-modulation of hypnotic drug use. Although the intention in prescribing a hypnotic may be to treat the confounding condition rather than to treat insomnia, to the extent that the causal pathway is mediated by hypnotic drug prescribing, the hypnotic drugs have a preventable causal role. This model also illustrates that hypnotic prescribing initiated independent of confounders (e.g., in treatment of 'primary' insomnia) may also influence drug use

diazepam, flunitrazapam, lorazepam, etc. that are commonly prescribed for sleep, a total of about 60 million visits with prescriptions for drugs often (not always) used as sleeping pills was arrived at. These estimates are murky, but the National Survey data seem to show that as much as 40-90\% of the times physicians prescribe hypnotics and related anxiolytics, they record no indication of insomnia or other recognized medical indication for the prescription. There is very little knowledge of why this might occur. The National Ambulatory Medical Care Survey is accessible contemporary documentation of how hypnotic drug prescriptions are written, but it is not alone. In the sparse literature available about why physicians prescribe hypnotics, there has been much evidence that there is often no articulated belief that the hypnotic prescribed is indicated for insomnia or a related sleep disturbance, and often the patient denies insomnia and does not know why the prescription was given $[19,20]$. Current electronic medical audit systems are increasingly encouraging physicians who wish to prescribe hypnotics to check off a diagnosis of insomnia, but no evidence has been located that checking off this diagnosis on a computer screen would reliably indicate that sleep symptoms had actually been discussed with the patient, much less that current criteria for insomnia disorder had been met. It is hard to estimate how often a hypnotic prescription was offered for a confounding indication and how often there was no medical indication at all.

There may be no authoritative documentation, but young hospital physicians are sometimes instructed to prescribe hypnotics so that they will not be awakened at 
night for requests for such as-needed prescriptions. In such cases, the hypnotic prescriptions are to allow the physicians to sleep, not the patients. Allow me to document that this was my training at prestigious hospitals. Physicians sometimes say that hospital night nurses insist that their patients have hypnotic prescriptions in hopes that they (the nurses) will have a less difficult night. Perhaps these prescriptions are so that they (the night nurses) can get some sleep. If these are the rationales for such prescriptions, this would not produce confounding of medical indications for hypnotics, but it is hard to exclude confounding risks related to caregiver fatigue. Such habits of automatic prescribing might carry over from hospitals into the outpatient arena.

There may be no published documentation, but physicians have repeatedly told me that they prescribe hypnotics in fear that unsatisfied patients will depart for new physicians. Physicians are fond of their patients, but it is difficult to exclude any financial motivation to retain patients. Unstated is the tendency for patients prescribed addicting drugs to return for refills to avoid withdrawal symptoms. Years ago, one of the country's most distinguished sleep physicians said he had surveyed a group of outstanding clinicians, who explained that in prescribing hypnotics, they selected patients whom they knew well and trusted. The described gift-giving also implied the use of hypnotics to maintain doctor-patient relationships rather than to treat sleep symptoms. It seems currently unknown if such attitudes are correlated with mortality risks, thus constituting a source of confounding by indication.

To summarize, almost all of the studies listed in Table 1 made some attempts to adjust for confounding by indication by adjusting for comorbidities and other risk factors that might have confounded the associations of hypnotics with mortality risk. The three studies emphasized in Sect. 4.5 planned very extensive efforts to adjust for comorbidities, and two used matching of cases to controls, but observed that final models with full adjustment of many covariates reduced the estimated risk ratios rather little. It is true that electronic-record comorbidity data may have had limited accuracy and precision, and there are too many small or uncommon sources of comorbidity risks to adjust for all of them, but if confounding by medical indication entirely explained the associations, greater success with the adjustments utilized might have been expected. Considering that many hypnotic prescriptions are not explained by documented medical indications, in such cases, the operative motivating indications for prescription are unknown and possibly pseudo-randomly inspired. It seems unlikely that such undocumented indications are strong mortality risk predictors, yet it is likewise impossible to exclude confounding by such mysterious unknown indications.

\subsection{Lack of Data for Cause and Time of Death}

An important limitation in almost all of the epidemiology about hypnotics risks is lack of data on the causes of death and times of death associated with hypnotics usage. Although such information is not essential to demonstrating the statistical significance of the hypnotics-mortality association, more information on causes of death would be helpful in understanding possible causal mechanisms by which hypnotics may increase mortality risks. A distinct pattern of causes of death would add to the evidence for causality. Moreover, it might be found that deaths of hypnotics users occur out-of-proportion at night, as some old data suggested [21]. If hypnotics use is associated with death at night, that would support the hypothesis that hypnotics may cause covert nocturnal compromise of respiration.

\subsection{Over-Adjustment of Confounders}

Use of hypnotics is associated with a wide variety of comorbidities $[9,10]$. Generally, it has been assumed that these comorbidities are confounding indications for hypnotics, as was discussed in Sect. 4.6. What is often not recognized is that hypnotics cause comorbidities such as insomnia, depression, infection, gastric regurgitation, and even perhaps cancer, as proven by industry-sponsored randomized controlled clinical trials [22-26]. Also, controlled trials prove that many hypnotics impair next-day driving performance. Moreover, hypnotics are epidemiologically associated with an increased rate of driver-at-fault vehicle crashes as well as falls and serious infections [13, 27-36]. Other studies show that hypnotic use is associated with new cancer incidence [37-39]. Such studies fall somewhat short of causal proof that hypnotics cause deaths from injuries, infections, suicide, and cancer, but for purposes of epidemiologic studies, it should be assumed that hypnotic prescribing may produce reverse causation of several forms of comorbidity, as suggested in Fig. 4.

That hypnotics can cause comorbidities raises problems in analysis of the association of hypnotics and mortality. To adjust for confounding of indications, it may seem desirable to adjust for comorbidities such as insomnia, depression, infection, and cancer. However, to the extent that such comorbidities are caused by the hypnotics, this would be over-adjustment. Over-adjustment may in effect assign estimated variance in mortality to comorbidities that are primarily caused by the hypnotics, leading to underestimation of mortality association that should be attributed to the hypnotics. Studies such as that of Jaussent et al., in which the association of hypnotic use and mortality lost significance once adjusted for depression, etc., might suffer from such over-adjustment and might obscure the true risk 


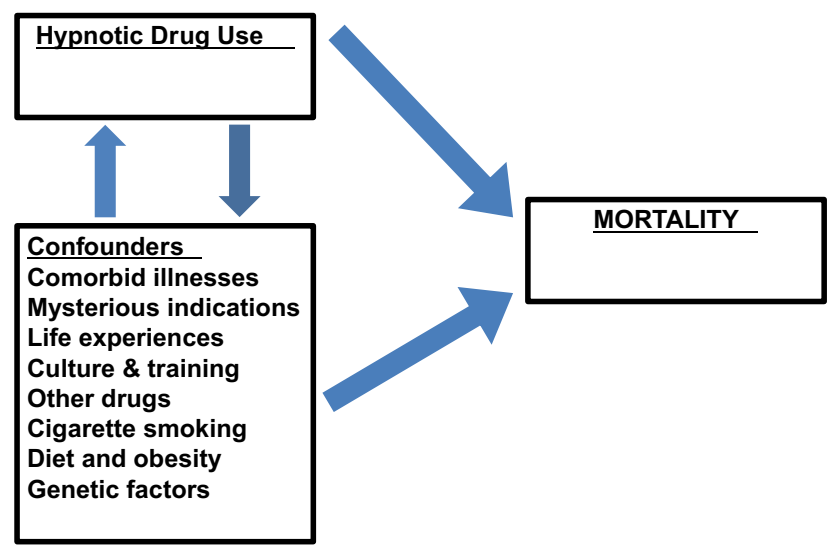

Fig. 4 A causal model in which bidirectional interactions occur between hypnotic drug prescribing and confounders. Examples of such bidirectional causal interactions would be those between hypnotics and depression or hypnotics and infections. In this model, there is a pathway of hypnotic drug prescribing mediating comorbid illness that may then have effects on mortality. In addition, as in Fig. 3, confounders may mediate mortality effects through influences upon hypnotic usage. It is doubtful that the causal pathways could be distinguished without some approach resembling path analysis with structural equation modeling

associated with hypnotics [5]. If the patients in the study have received hypnotics before the baseline assessment, this prior usage before the time of baseline assessment may also confound true hypnotic effects with the comorbidities that have already resulted from hypnotics.

Over-adjustment of comorbidities, excessively long prospective follow-ups with overlap between hypnotic-user groups and control groups in terms of hypnotics consumption during follow-up, inadequate specification of when the drugs studied are true hypnotics, and inadequate specification of drug dosage consumed may all lead to underestimation of the true association of hypnotics with mortality risks.

\subsection{Limitations due to Genetic Effects and New Research Possibilities}

Genetic polymorphisms probably influence hypnotics consumption, either through causing insomnia and circadian rhythm sleep disorders or through other influences on hypnotics consumption. Perhaps hypnotics consumption is modified by genetic metabolic alterations analogous to effects of alcohol dehydrogenase polymorphisms on alcoholism [40, 41]. There is a heritable influence upon insomnia, but this has not been distinguished from possible heritable effects upon hypnotic usage [42, 43]. As yet, polymorphisms influencing hypnotics consumption independent of insomnia and depression have not been recognized.
If polymorphisms influence hypnotic consumption and independently influence mortality (e.g., see Fig. 2 model), then genetic influences could confound estimates of the association of hypnotic usage and mortality.

From a more scientifically hopeful point of view, genetic factors have potential to clarify the causal component of hypnotic effects on mortality. Consider the more complete (and most probable) causal model that is illustrated in Fig. 5. Note that Fig. 5 does not necessarily imply that the same polymorphisms influence hypnotic drug use and directly influence mortality. There may be genetic polymorphisms that only influence mortality mediated through influences on hypnotics consumption. Genetic factors are unique from other potential confounders in that their causality pathways are one-way. Neither hypnotic usage nor other confounders alter genetic polymorphisms. Rather, if a sample has racially homogeneous ancestry and at least some of the population are heterozygous for a genetic polymorphism, the presence of that polymorphism in offspring may be regarded as randomly determined. This is the basis of Mendelian randomization analyses that consider genetic effects on an instrumental variable (e.g., hypnotics consumption) and an outcome variable (e.g., mortality) in order to estimate the component size of the causal pathway leading from genotype to hypnotics consumption to modified mortality [44-47]. Mendelian randomization eliminates the interference of most confounders in estimating causality, though there remain various complex issues that must be considered. It now seems feasible to perform such causality studies, but one does not know when results might be forthcoming.

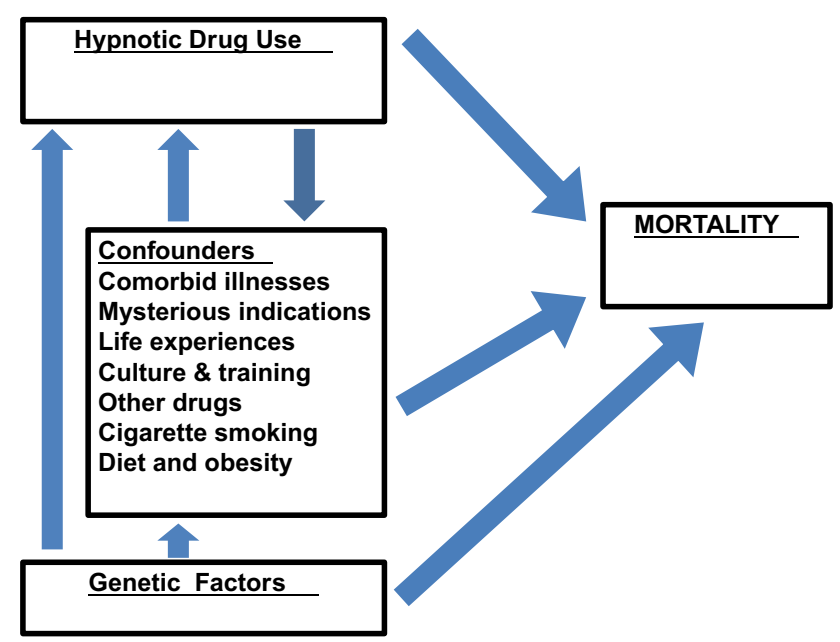

Fig. 5 Revises the model in Fig. 4 to emphasize that genetic factors may influence confounders such as comorbid illnesses or cigarette smoking and cause mortality indirectly through these pathways, as well as through what may be conceived as direct effects on mortality. However, in general, neither hypnotic drugs nor confounders such as comorbid illnesses or life experiences can influence our genetic inheritance 


\section{Discussion}

\subsection{The Causality of Hypnotics Effects on Mortality}

It has been amply demonstrated that hypnotics consumption predicts increased future mortality. Although the 34 studies in Table 1 are heterogeneous in the limitations that may lead to overestimation and underestimation of true causes of risk (which may explain the high variability of observed risk ratios), there was a persuasive consensus that hypnotics risk ratios were positive. Given the many limitations of epidemiologic studies demonstrating associations of hypnotics consumption with mortality risks, with several possibilities for overestimation and underestimation of the risks attributable to hypnotics, it can be argued that no epidemiologic method relying on clinical observation alone can provide accurate scientific assessment of the mortality caused by hypnotics. Since some of the best-adjusted studies yielded among the highest risk ratios [9, 10, 15], it appears that limitations tending towards underestimation of hypnotics risk often over-balanced the limitations tending towards overestimation. It seems unlikely (but not implausible) that so much mortality risk could be entirely explained by unknown confounders.

Of course, that hypnotics cause some small percentage of deaths is not really open to question. Recently, several US jurisdictions have concluded that injecting hypnotic drugs are among the preferred methods of executing condemned prisoners [48]. Drugs that have been used as hypnotics such as barbiturates and midazolam have been employed. Barbiturates have for decades been a preferred method of euthanizing experimental animals [48, 49]. For these applications, the mechanisms by which hypnotics hyperpolarize and inhibit respiratory neurons leading to death are well understood at the molecular, cellular, physiologic and organismic levels. Also reported are thousands of overdose deaths per year attributable to hypnotics, with a larger number of cases in which hypnotics as well as opiates and other sedatives are lethal in combination [50-52]. It has been suggested that currently, tens of thousands of yearly self-injury deaths are related to hypnotics and sedative use [53].

In addition to recognized overdoses, it is likely that many hypnotics-induced deaths may be unrecognized and under-reported. Because most patients who die unexpectedly at night are not autopsied, or because adequate assays of all hypnotics present at death are not available, or too much time for post-mortem drug metabolism and degradation has occurred, the overdose may not be recognized. Normal approved hypnotic dosages might even be lethal under specific circumstances. Although the lethal dosage of benzodiazepine agonists is usually as much as hundreds of hypnotic doses, it drops by an extraordinary degree after moderate ingestions of alcohol $[54,55]$. Combinations of narcotics and hypnotics, especially with the addition of alcohol and other sedatives, may slow breathing [56]. There are no established safe dose ranges for combinations of hypnotics, sedatives, and narcotics. If an obese, elderly patient receiving accepted doses of hypnotics and opiates dies unexpectedly at night, how could a physician document that the drugs were responsible? Even when a patient dies under observation with respiratory and cardiac monitoring, it may be difficult to demonstrate that an unintended drug-induced suppression of respiration led to the terminal event. Using data given in the supplement to reference [10], it can be estimated that only one death per 5000 hypnotic doses consumed would suffice to cause the hundreds of thousands of US deaths associated with hypnotics. Is it not plausible that such a tiny rate of lethal outcomes could occur? Even when a physician signing a death certificate suspects that hypnotics may have played a role, the physician usually lacks proof and may be reluctant to attribute the death to a drug that the physician may have prescribed. It is easier to attribute the death to some natural comorbid process for which nobody is responsible, leading to under-reporting of hypnotics-caused deaths.

\subsection{Bradford Hill Criteria of Causation are Fulfilled}

Review of the nine Bradford Hill criteria of causation support the hypothesis of hypnotics causing excess mortality. (1) The strength of association is strong, especially in recent studies that utilize electronic records data identifying hypnotic prescriptions. (2) Consistency is the strongest evidence, with 33 of 34 studies showing a positive association of hypnotic consumption and mortality. (3) Several kinds of specificity of hypnotics-mortality associations have been identified: the excess of deaths at night among hypnotics users [21], the excesses of infection [13], COPD [57], and suicide deaths [50], and the excess of specific cancers such as lung and esophagus [38]. (4) The temporality of hypnotic usage preceding death is consistent with causation. (5) The significant dose-response data are consistent with causation [9]. (6) Causality is plausible, because the mechanisms of benzodiazepine-agonist suppression of respiration are well understood, hypnotic effects causing depression which causes suicide are understood, immune effects of these drugs make infection plausible, and clastogenicity and immune interference make cancer plausible. (7) The analogies between use of hypnotics for executions and euthanasia, the Medical Examiner determinations of overdose deaths, and the larger picture of hypnotic-associated mortality are highly coherent. (8) Experimental studies such as laboratory experi- 
ments demonstrating hypnotics causing overdose deaths in animals, and laboratory experiments causing infections and cancers in animals, and so forth, provide strong evidence. (9) The strong analogies of hypnotics-associated mortality with cigarette-associated mortality, alcoholism-associated mortality, and other addictive drug lethalities support the lethality of hypnotics. Despite how completely the evidence fulfills the Bradford Hill criteria for epidemiologic proof of causation, the impossibility of ruling out all confounders of the hypnotics-mortality association has led to popular demand for stronger evidence that can probably only be derived from other approaches.

\subsection{What is Needed for Augmented Scientific Conclusiveness?}

To obtain scientifically conclusive assessments of mortality risks posed by hypnotics, there are two scientific strategies available. The first is the Mendelian randomization strategy discussed in Sect. 4.9 that may become increasingly feasible as more and more electronic medical records contain both hypnotic drug administration data and patient genotypes. However, a Mendelian randomization study of hypnotic risks would require identification of genetic polymorphisms that (1) predispose towards increased or decreased hypnotics consumption, and (2) do not influence mortality through pathways independent of their effects on hypnotics consumption. It is not yet known whether such polymorphisms can be identified. The alternative is the classic gold standard of causal proof: randomized controlled clinical trials.

Unfortunately, there are no randomized placebo-controlled clinical trials of hypnotics in mortality-susceptible samples large enough and long enough to determine with reasonable statistical power whether hypnotics cause excess mortality. Industry trials have avoided the most mortality-vulnerable part of the population who receive hypnotics. Several studies have suggested that hypnotics are associated with a magnitude of mortality risks comparable to that of cigarettes $[3,10,15]$. Nevertheless, recognize that no cigarette manufacturers have attempted large clinical trials hoping to demonstrate that their products are without serious mortality risk. Likewise, no hypnotics manufacturer has attempted a large clinical trial intended to demonstrate that their hypnotic is without serious mortality risk. The reasons would be anybody's guess, assuming the manufacturers believe that their products would be proven safe. A prestigious medical journal published one light-hearted commentary suggesting that the problem might be similar to our lack of controlled studies concerning the consequences of jumping out of airplanes without parachutes [58]. This may imply an ethical difficulty in performing studies of lethal risks, but it is ethical (with informed consent) to randomize patients to hypnotic drugs or placebo under conditions when the participants would be prescribed the drug on clinical grounds outside of research. Such large drug studies are frequently done in cardiology among vulnerable patients. In contrast, it is unethical not to randomize and to determine what the risk is that millions currently undergo. Such studies have now become ethically obligatory. Apart from the classic double-blind randomized drug administration trial, there are interesting new ethical approaches to randomly modifying hypnotic consumption among large groups of patients [59], though such an approach would seem difficult to implement double-blind. Unfortunately, clinical medicine may wait a long time before any investigator finds a sponsor for such a study unless the regulatory authorities take their responsibility to protect the public seriously enough to insist that manufacturers perform adequate mortality-risk studies.

\subsection{What is Recommended Awaiting Immaculate Evidence of How Much of Hypnotic Mortality Risks are Causal?}

There is conclusive evidence that use of hypnotics is associated with substantially increased mortality in the population. There is conclusive proof that hypnotic drugs are lethal in overdoses and in animals. What should be done while no scientifically conclusive estimate of the population causal mortality risk of hypnotics is available? Remember, there is no indication whatsoever that hypnotics used for sleep ever save lives. In medicine, a physician must use the best data available to make clinical decisions, even when complete evidence is lacking, and even when one might wish there were a better evidence base.

The best example of how medical experts should act is the belated medical leadership on cigarettes. Several studies have suggested that the population mortality risk associated with hypnotics is quite comparable or greater than that of cigarettes, whereas other studies have suggested that the hypnotic-associated risk could be somewhat smaller [3, 10, 15]. The American Cancer Society's Cancer Prevention Study II (CPSII, previously discussed) is an important example, because until quite recently, US public health authorities and United Nations authorities considered CPSII as providing the most authoritative estimate of population cigarette mortality causality. As discussed, in one analysis of CPSII data, the risk of hypnotics was rather similar to that of cigarettes when considered simultaneously in the very same Cox proportional hazards models using exactly the same data sampling methods and covariate adjustments [3]. There are no randomized controlled trials adequately proving that cigarettes cause 
deaths, but the epidemiologic data of CPSII and many supporting studies have been considered sufficient evidence for physicians to completely avoid recommending cigarettes. Considering that hypnotics are favorite drugs for euthanizing animals and executing prisoners, cigarettes being unsatisfactory for these purposes, why not conclude that hypnotics likely carry mortality risks close to the same order of magnitude as cigarettes? Why not rely on the same CPSII evidence to take similar action to limit hypnotic consumption?

Official US FDA-approved prescribing information lists all kinds of risks of hypnotics for which the evidence is laughably weak compared with the evidence regarding mortality. The mortality risks of hypnotics should be listed. There should be warnings on the bottles. Are 24 significant positive studies not enough? Until there is better evidence of safety, the reasonable medical procedure is not to wait forever but to act. The reasonable procedure would be to restrict hypnotic drugs to controlled trials and to compassionate hospice use - unless and until such hypnotics might be proven safe in Mendelian randomization studies or in randomized placebo-controlled trials.

For general clinical use as hypnotics, the risks outweigh benefits for benzodiazepine agonists and diphenhydramine used as hypnotics. The risk of killing patients outweighs any benefits. These drugs should not be prescribed as hypnotics except in further clinical trials and hospice situations. In general, it appears that cognitive behavioral treatment of insomnia [60] and bright light treatment for circadian rhythm disorders linked to insomnia [61] are the safest and probably also the most effective treatments for insomnia. Cognitive behavioral treatment of insomnia or bright light, often used in combination, should be the first choice. For trazodone, doxepin, and melatonin when used for sleep, there are no adequate data to estimate whether long-term risks outweigh benefits. European authorities concluded that the benefits/risks ratio of melatonin is probably better than that of ramelteon, a melatonin agonist [19], so there is no reason to use ramelteon. If a decision is made that some medication for insomnia must be risked, it appears that trazodone in minimal doses, doxepin (in low dosage, e.g., $6 \mathrm{mg}$ ), or melatonin would be the least-risky options currently available, and when used, these drugs should generally be combined with cognitive behavioral treatment or bright light treatment.

\section{Conclusion}

A remarkable 33 of 34 epidemiologic studies have found an elevated mortality risk among hypnotics users. That some component of the associated deaths is causal is not open to question because of Medical Examiner-verified overdose deaths. Hypnotics may also cause unrecognized respiratory deaths due to combinations of hypnotics with narcotics, other sedatives, alcohol, obesity, age-related sleep apnea, and other comorbidities. Hypnotics further cause accidents, infections, depression, and possibly cancers, which may lead to death. The evidence of mortality risk among the best-studied hypnotics is strong enough to restrict these drugs to hospice care, though the manufacturers should be permitted to conduct randomized placebocontrolled trials with patients with high or moderate mortality risk designed to prove (if possible) that the hypnotics are safe. Otherwise, since manufacturers have so far declined their option to do large mortality-safety trials, and it is difficult to exclude any confounders influencing epidemiologic association of hypnotic usage with death ${ }^{1}$, further absolute proof of causality may await Mendelian randomization studies.

\section{Compliance with Ethical Standards}

Funding No sources of funding were used to assist in the preparation of this study.

Conflicts of interest Daniel Kripke is a long-time critic of hypnotic drugs, especially their adverse effects, in many publications and the non-profit website http://www.DarkSideOfSleepingPills.com. The author's family owns stock and options in a large conglomerate that secondarily holds $1-2 \%$ of its capital in Sanofi stock, over which the author has no control.

Contribution to authorship Dr. Kripke wrote this manuscript without assistance. He thanks anonymous reviewers for helpful comments.

\section{References}

1. Hammond EC. Some preliminary findings on physical complaints from a prospective study of 1,064,004 men and women. Am J Public Health. 1964;54:11-24.

2. Kripke DF, Simons RN, Garfinkel L, Hammond EC. Short and long sleep and sleeping pills: Is increased mortality associated? Arch Gen Psychiatry. 1979;36(1):103-16.

3. Kripke DF, Klauber MR, Wingard DL, Fell RL, Assmus JD, Garfinkel L. Mortality hazard associated with prescription hypnotics. Biol Psychiatry. 1998;43(9):687-93.

4. Kripke DF, Garfinkel L, Wingard DL, Klauber MR, Marler MR. Mortality associated with sleep duration and insomnia. Arch Gen Psychiatry. 2002;59(2):131-6.

5. Jaussent I, Ancelin ML, Berr C, Peres K, Scali J, Besset A, et al. Hypnotics and mortality in an elderly general population: a 12-year prospective study. BMC Med. 2013;11(1):212.

6. NIOM. Sleeping pills, insomnia, and medical practice. Washington, DC: National Academy of Sciences; 1979.

\footnotetext{
${ }^{1}$ For more evidence of the need for clinical trials adequate to assess the mortality risks of hypnotics, see the Dr. Kripke's Citizen Petition to the FDA which will be available by searching the FDA Docket at http://www.regulations. gov/\#! searchResults;rpp $=25 ; \mathrm{po}=0 ; \mathrm{s}=$ citizen $\%$ 252Bpetition\%252BKripke;fp=true;ns=true.
} 
7. National Institutes of Health State of the Science. Conference statement on manifestations and management of chronic insomnia in adults, June 13-15, 2005. Sleep. 2005;28(9):1049-57.

8. Saras A, Gisselmann G, Vogt-Eisele AK, Erlkamp KS, Kletke O, Pusch $\mathrm{H}$, et al. Histamine action on vertebrate GABAA receptors: direct channel gating and potentiation of GABA responses. J Biol Chem. 2008;283(16):10470-5.

9. Weich S, Pearce HL, Croft P, Singh S, Crome I, Bashford J, et al. Effect of anxiolytic and hypnotic drug prescriptions on mortality hazards: retrospective cohort study. BMJ. 2014;348:g1996.

10. Kripke DF, Langer RD, Kline LE. Hypnotics' association with mortality or cancer: a matched cohort study. BMJ Open. 2012;2(1):e000850.

11. Fox C, Richardson K, Maidment ID, Savva GM, Matthews FE, Smithard D, et al. Anticholinergic medication use and cognitive impairment in the older population: the medical research council cognitive function and ageing study. $\mathrm{J}$ Am Geriatr Soc. 2011;59(8):1477-83.

12. Kojima M, Wakai K, Kawamura T, Tamakoshi A, Aoki R, Lin Y, et al. Sleep patterns and total mortality: a 12-year follow-up study in Japan. J Epidemiol. 2000;10(2):87-93.

13. Obiora E, Hubbard R, Sanders RD, Myles PR. The impact of benzodiazepines on occurrence of pneumonia and mortality from pneumonia: a nested case-control and survival analysis in a population-based cohort. Thorax. 2012;68(2):163-70.

14. Neutel CI, Johansen HL. Association between hypnotics use and increased mortality: causation or confounding? Eur J Clin Pharmacol. 2015;71(5):637-42.

15. Mallon L, Broman JE, Hetta J. Is usage of hypnotics associated with mortality? Sleep Med. 2009;10(3):279-86.

16. Belleville G. Mortality hazard associated with anxiolytic and hypnotic drug use in the national population health survey. Can $\mathbf{J}$ Psychiatry. 2010;55(9):558-67.

17. Chen H-C, Su T-P, Chou P. A 9-year follow-up study of sleep patterns and mortality in community-dwelling older adults in Taiwan. Sleep. 2013;36(8):1187-98.

18. Ford ES, Wheaton AG, Cunningham TJ, Giles WH, Chapman DP, Croft JB. Trends in outpatient visits for insomnia, sleep apnea, and prescriptions for sleep medications among US adults: findings from the National Ambulatory Medical Care Survey 1999-2010. Sleep. 2014;37(8):1283-93.

19. Bertisch SM, Herzig SJ, Winkelman JW, Buettner C. National use of prescription medications for insomnia: NHANES 1999-2010. Sleep. 2014;37(2):343-9.

20. Mellinger GD, Balter MB, Uhlenhuth EH. Insomnia and its treatment. Prevalence and correlates. Arch Gen Psychiatry. 1985;42:225-32.

21. Kripke DF, Garfinkel L. Excess nocturnal deaths related to sleeping pill and tranquilliser use. Lancet. 1984;I(8368):99.

22. Gagliardi GS, Shah AP, Goldstein M, Denua-Rivera S, Doghramji K, Cohen S, et al. Effect of zolpidem on the sleep arousal response to nocturnal esophageal acid exposure. Clin Gastroenterol Hepatol. 2009;7(9):948-52.

23. Kripke DF. Hypnotics cause insomnia: evidence from clinical trials. Sleep Med. 2014;15(9):1168-9.

24. Kripke DF. Greater incidence of depression with hypnotics than with placebo. BMC Psychiatry. 2007;7:42.

25. Joya FL, Kripke DF, Loving RT, Dawson A, Kline LE. Metaanalyses of hypnotics and infections: eszopiclone, ramelteon, zaleplon, and zolpidem. J Clin Sleep Med. 2009;5(4):377-83.

26. Kripke DF. Possibility that certain hypnotics might cause cancer in skin. J Sleep Res. 2008;17(3):245-50.

27. Verster JC, Veldhuijzen DS, Patat A, Olivier B, Volkerts ER. Hypnotics and driving safety: meta-analyses of randomized controlled trials applying the on-the-road driving test. Curr Drug Saf. 2006;1(1):63-71.
28. Verster JC, Roth T. Drivers can poorly predict their own driving impairment: a comparison between measurements of subjective and objective driving quality. Psychopharmacology (Berl). 2011;219(3):775-81.

29. Vermeeren A, Sun H, Vuurman EF, Jongen S, Van Leeuwen CJ, Van Oers AC, et al. On-the-road driving performance the morning after bedtime use of suvorexant 20 and $40 \mathrm{mg}$ : a study in nonelderly healthy volunteers. Sleep. 2015. (Epub ahead of print).

30. Hansen RN, Boudreau DM, Ebel BE, Grossman DC, Sullivan SD. Sedative hypnotic medication use and the risk of motor vehicle crash. Am J Public Health. 2015;105(8):e64-9. doi:10. 2105/AJPH.2015.302723.

31. Philip P, Chaufton C, Orriols L, Lagarde E, Amoros E, Laumon B, et al. Complaints of poor sleep and risk of traffic accidents: a population-based case-control study. PLoS One. 2014;9(12):e114102. doi:10.1371/journal.pone.0114102.

32. Orriols L, Philip P, Moore N, Castot A, Gadegbeku B, Delorme $\mathrm{B}$, et al. Benzodiazepine-like hypnotics and the associated risk of road traffic accidents. Clin Pharmacol Ther. 2011;89(4):595-601.

33. Diem SJ, Ewing SK, Stone KL, Ancoli-Israel S, Redline S, Ensrud KE. Use of non-benzodiazepine sedative hypnotics and risk of falls in older men. J Gerontol Geriatr Res. 2014;3(3):158.

34. Berry SD, Lee Y, Cai S, Dore DD. Nonbenzodiazepine sleep medication use and hip fractures in nursing home residents. JAMA Intern Med. 2013;173(9):754-61.

35. Kolla BP, Lovely JK, Mansukhani MP, Morgenthaler TI. Zolpidem is independently associated with increased risk of inpatient falls. J Hosp Med. 2013;8(1):1-6.

36. Huang CY, Chou FH, Huang YS, Yang CJ, Su YC, Juang SY, et al. The association between zolpidem and infection in patients with sleep disturbance. J Psychiatr Res. 2014;54(7):116-20.

37. Kao CH, Sun LM, Su KP, Chang SN, Sung FC, Muo CH, et al. Benzodiazepine use possibly increases cancer risk: a populationbased retrospective cohort study in Taiwan. J Clin Psychiatry. 2012;73(4):e555-60. doi:10.4088/JCP.11m07333.

38. Kao CH, Sun LM, Liang JA, Chang SN, Sung FC, Muo CH. Relationship of zolpidem and cancer risk: a Taiwanese population-based cohort study. Mayo Clin Proc. 2012;87(5):430-6.

39. Sivertsen B, Salo P, Pentti J, Kivimaki M, Vahtera J. Use of sleep medications and risk of cancer: a matched case-control study. Sleep Med. 2015. doi:10.1016/j.sleep.2015.05.003. (Epub ahead of print).

40. Lawlor DA, Nordestgaard BG, Benn M, Zuccolo L, TybjaergHansen A, Davey SG. Exploring causal associations between alcohol and coronary heart disease risk factors: findings from a Mendelian randomization study in the Copenhagen General Population Study. Eur Heart J. 2013;34(32):2519-28.

41. Au Yeung SL, Jiang C, Cheng KK, Liu B, Zhang W, Lam TH, et al. Is aldehyde dehydrogenase 2 a credible genetic instrument for alcohol use in Mendelian randomization analysis in Southern Chinese men? Int J Epidemiol. 2013;42(1):318-28.

42. Hublin C, Partinen M, Koskenvuo M, Kaprio J. Heritability and mortality risk of insomnia-related symptoms: a genetic epidemiologic study in a population-based twin cohort. Sleep. 2011;34(7):957-64.

43. Wing YK, Zhang J, Lam SP, Li SX, Tang NL, Lai KY, et al. Familial aggregation and heritability of insomnia in a community-based study. Sleep Med. 2012;13(8):985-90.

44. Boef AG, Dekkers OM, le Cessie S. Mendelian randomization studies: a review of the approaches used and the quality of reporting. Int J Epidemiol. 2015;44(2):496-511.

45. Davey Smith G, Hemani G. Mendelian randomization: genetic anchors for causal inference in epidemiological studies. Hum Mol Genet. 2014;23(R1):R89-98.

46. Burgess S, Daniel RM, Butterworth AS, Thompson SG. Network Mendelian randomization: using genetic variants as instrumental variables to investigate mediation in causal pathways. Int J Epidemiol. 2015;44(2):484-95. 
47. Thanassoulis G. Mendelian randomization: how genetics is pushing the boundaries of epidemiology to identify new causes of heart disease. Can J Cardiol. 2012;29(1):30-6.

48. Lopez-Munoz F, Ucha-Udabe R, Alamo C. The history of barbiturates a century after their clinical introduction. Neuropsychiatr Dis Treat. 2005;1(4):329-43.

49. Leary S, Members of Panel. AVMA Guidelines for the Euthanasia of Animals: 2013 Edition. 2013.0.1 ed. Schaumburg:American Veterinary Medical Association;2013.

50. Park TW, Saitz R, Ganoczy D, Ilgen MA, Bohnert AS. Benzodiazepine prescribing patterns and deaths from drug overdose among US veterans receiving opioid analgesics: case-cohort study. BMJ. 2015;350:h2698. doi:10.1136.bmj.h2698.

51. Jann M, Kennedy WK, Lopez G. Benzodiazepines: a major component in unintentional prescription drug overdoses with opioid analgesics. J Pharm Pract. 2014;27(1):5-16.

52. Cooper JR. Sedative-hypnotic drugs: risks and benefits. Rockville: U.S. Department of HEW, National Inst. on Drug Abuse; 1977.

53. Rockett IR. Self-injury is the eighth leading cause of death in the United States: it is time to pay attention. JAMA Psychiatry. 2015;72(11):1069-70. doi:10.1001/jamapsychiatry.2015.1418.

54. Nobody. The DAWN report: Benzodiazepines in combination with opioid pain relievers or alcohol: greater risk of more serious ED visit outcomes. DAWN-192. Rockville:Substance Abuse and Mental Health Services Administration, Center for Behavioral Health Statistics and Quality;2014.

55. Okamoto M, Rao SN, Aaronson LM, Walewski JL. Ethanol drug interaction with chlordiazepoxide and pentobarbital. Alcohol Clin Exp Res. 1985;9(6):516-21.

56. Webster LR, Choi Y, Desai H, Webster L, Grant BJ. Sleepdisordered breathing and chronic opioid therapy. Pain Med. 2008;9(4):425-32.

57. Chung WS, Lai CY, Lin CL, Kao CH. Adverse respiratory events associated with hypnotics use in patients of chronic obstructive pulmonary disease: a population-based case-control study. Medicine (Baltimore). 2015;94(27):e1110.

58. Smith GC, Pell JP. Parachute use to prevent death and major trauma related to gravitational challenge: systematic review of randomised controlled trials. BMJ. 2003;327(7429):1459-61.

59. Tannenbaum C, Martin P, Tamblyn R, Benedetti A, Ahmed S. Reduction of inappropriate benzodiazepine prescriptions among older adults through direct patient education: the EMPOWER cluster randomized trial. JAMA Intern Med. 2014;174(6):890-8.

60. Mitchell MD, Gehrman P, Perlis M, Umscheid CA. Comparative effectiveness of cognitive behavioral therapy for insomnia: a systematic review. BMC Fam Pract. 2012;13(1):40.

61. Lack L, Wright H, Paynter D. The treatment of sleep onset insomnia with bright morning light. Sleep Biol Rhythms. 2007;3:173-9.

62. Rumble R, Morgan K. Hypnotics, sleep, and mortality in elderly people. J Am Geriatr Soc. 1992;40:787-91.

63. Isacson D, Carsjo K, Bergman U, Blackburn JL. Long-term use of benzodiazepines in a Swedish community: an eight-year follow-up. J Clin Epidemiol. 1992;45(4):429-36.

64. Thorogood M, Cowen P, Mann J, Murphy M, Vessey M. Fatal myocardial infarction and use of psychotropic drugs in young women. Lancet. 1992;340:1067-8.

65. Brabbins CJ, Dewey ME, Copeland RM, Davidson IA, McWilliam C, Saunders P, et al. Insomnia in the elderly: prevalence, gender differences and relationships with morbidity and mortality. Int J Ger Psych. 1993;8:473-80.

66. Merlo J, Hedblad B, Ogren M, Ranstam J, Ostergren PO, Ekedahl $\mathrm{A}$, et al. Increased risk of ischaemic heart disease mortality in elderly men using anxiolytics-hypnotics and analgesics. Eur $\mathbf{J}$ Clin Pharmacol. 1996;49:261-5.
67. Hays JC, Blazer DG, Foley DJ. Risk of napping: excessive daytime sleepiness and mortality in an older community population. J Am Geriatr Soc. 1996;44:693-8.

68. Sundquist J, Ekedahl A, Johansson S-E. Sales of tranquillizers, hypnotics/sedatives and antidepressants and their relationship with underprivileged area score and mortality and suicide rates. Eur J Clin Pharmacol. 1996;51:105-9.

69. Merlo J, Ostergren PO, Mansson NO, Hanson BS, Ranstam J, Blennow G, et al. Mortality in elderly men with low psychosocial coping resources using anxiolytic-hypnotic drugs. Scand J Public Health. 2000;28(4):294-7.

70. Hedner J, Caidahl K, Sjoland H, Karlsson T, Herlitz J. Sleep habits and their association with mortality during 5-year followup after coronary artery bypass surgery. Acta Cardiol. 2002;57(5): $341-8$.

71. Mallon L, Broman J-E, Hetta J. Sleep complaints predict coronary artery disease mortality in males: a 12-year follow-up study of a middle-aged Swedish population. J Int Med. 2002;251: 207-16.

72. Ahmad R, Bath PA. Identification of risk factors for 15-year mortality among community-dwelling older people using Cox regression and a genetic algorithm. J Gerontol A Biol Sci Med Sci. 2005;60A:1052-8.

73. Phillips B, Mannino DM. Does insomnia kill? Sleep. 2005; 28(8):965-71.

74. Lack LC, Prior K, Luszcz M. 708. Does insomnia kill the elderly? Sleep. 2006;29(Abstract Supplement):A240.

75. Fukuhara S, Green J, Albert J, Mihara H, Pisoni R, Yamazaki S, et al. Symptoms of depression, prescription of benzodiazepines, and the risk of death in hemodialysis patients in Japan. Kidney Int. 2006;70(10):1866-72.

76. Hausken AM, Skurtveit S, Tverdal A. Use of anxiolytic or hypnotic drugs and total mortality in a general middle-aged population. Pharmacoepidemiol Drug Saf. 2007;16(8):913-8.

77. Hoffmann VP, Dossenbach M, West TM, Lowry AJ. Mortality in a cohort of outpatients with schizophrenia: 3-year outcomes from the Intercontinental Outpatient Health Outcomes Study (IC-SOHO). Biol Psychiatry. 2007;61(8S):163S-164S. (Abstract).

78. Hublin C, Partinen M, Koskenvuo M, Kaprio J. Sleep and mortality: a population-based 22-year follow-up study. Sleep. 2007;30(10):1245-53.

79. Winkelmayer WC, Mehta J, Wang PS. Benzodiazepine use and mortality of incident dialysis patients in the United States. Kidney Int. 2007;72(11):1388-93.

80. Rod NH, Vahtera J, Westerlund H, Kivimaki M, Zins M, Goldberg $\mathrm{M}$, et al. Sleep disturbances and cause-specific mortality: results from the GAZEL cohort study. Am J Epidemiol. 2010;173(3):300-9.

81. Gisev N, Hartikainen S, Chen TF, Korhonen M, Bell JS. Mortality associated with benzodiazepines and benzodiazepine-related drugs among community-dwelling older people in Finland: a population-based retrospective cohort study. Can J Psychiatry. 2011;56(6):377-81.

82. Hartz A, Ross JJ. Cohort study of the association of hypnotic use with mortality in postmenopausal women. BMJ Open. 2012;2. doi:10.1136/bmjopen-2012-001413.

83. Frandsen R, Baandrup L, Kjellberg J, Ibsen R, Jennum P. Increased all-cause mortality with psychotropic medication in Parkinson's disease and controls: a national register-based study. Parkinsonism Relat Disord. 2014;20(11):1124-8.

84. Weisberg DF, Gordon KS, Barry DT, Becker WC, Crystal S, Edelman EJ, et al. Long-term prescription of opioids and/or benzodiazepines and mortality among HIV-infected and uninfected patients. J Acquir Immune Defic Synd. 2015;69(2):223-33. 
85. Pinot J, Herr M, Robine JM, Aegerter P, Arvieu JJ, Ankri J. Does the prescription of anxiolytic and hypnotic drugs increase mortality in older adults? J Am Geriatr Soc. 2015;63(6):1263-5.

86. Nakafero G, Sanders RD, Nguyen-Van-Tam JS, Myles PR. Association between benzodiazepine use and exacerbations and mortality in patients with asthma: a matched case-control and survival analysis using the United Kingdom Clinical Practice Research Datalink. Pharmacoepidemiol Drug Saf. 2015;24(8):793-802. 